\title{
Emergency department use of monoclonal antibody therapy in high risk COVID positive patients
}

\author{
Andrew R. Barbera, Kayla Wilson, James D. Melton III, Fred Blind, Donna M. Bhisitkul, Diana Degroot, Donna \\ Faviere, Joanne Fuell, Hal Escowitz, Timothy J Regan \\ Lakeland Regional Health, United States
}

Received: February 18, 2021

Accepted: March 21, 2021

Online Published: April 20, 2021

DOI: $10.5430 /$ jha.v10n2p38

URL: https://doi.org/10.5430/jha.v10n2p38

\begin{abstract}
Background: There have been many perceived barriers to the implementation of the mass use of monoclonal antibody therapy following the Food and Drug Administration's Emergency Use Authorization in November 2020. These barriers include identifying eligible patients, physical resources including trained staff members, space, and materials for the administration away from others to reduce transmission, and cost of the resources. However, Lakeland Regional Health was able to create a safe and efficient protocol to administer Bamlanivimab in the treatment of high risk COVID positive patients and initiate this proposed pathway within 24 hours of receipt of the first shipment of medication.

Methods: Critical to the development and success of this protocol was a multi-disciplinary approach focused on identifying and utilizing preexisting resources to ensure safe and efficient administration of this treatment to as many eligible patients as possible. Another crucial aspect was the utilization of the emergency department providers for identifying high risk eligible patients and as a safe and effective treatment setting.

Results: This article is intended to demonstrate a best practice pathway to identify and administer Bamlanivimab, or similar treatments, and will not discuss outcomes or efficacy of the medication. To date Lakeland Regional Health has successfully treated over 1,000 high risk COVID-19 positive patients within our community.

Conclusions: By identifying and utilizing similar resources and pathways available at individual medical centers, it is possible to safely and efficiently treat high risk COVID positive patients with monoclonal antibody therapy on a large scale.
\end{abstract}

Key Words: COVID, COVID-19, Coronavirus, Monoclonal antibody therapy, COVID-19 treatment, Bamlanivimab, Emergency Department

\section{INTRODUCTION}

Lakeland Regional Health (LRH), an 864-bed comprehensive tertiary referral hospital with a large catchment area, was designated by the Florida Department of Health to receive the first weekly allocation of Bamlanivimab in early November 2020. Bamlanivimab, a recombinant monoclonal antibody, received Emergency Use Authorization (EUA) from the Food and Drug Administration(FDA) for the outpatient treatment of mild to moderate COVID-19 infected patients that met the high risk criteria. As part of this effort and to best serve our community, LRH needed to develop a procedure to identify high risk COVID positive patients who would benefit from this medication. LRH then needed to further develop a protocol and system to administer the monoclonal antibody as safely and efficiently as possible. This was accomplished successfully by utilizing a multi-disciplinary approach and identifying and adapting existing resources.

Within 24 hours of receiving the medication we were able to

*Correspondence: Andrew R. Barbera; Email: barbera.andrew @ gmail.com; Address: Lakeland Regional Health, United States. 
have a protocol in place, identify patients who qualify, and administer the very first treatment. The protocol we have created has proven a reliable and efficient pathway to identify and treat our high risk COVID-19 positive patient population with 900 patients infused with Bamlanivimab within the first three months of treatment availability. This article is intended only to demonstrate a best practice pathway to identify and administer Bamlanivimab or similar treatments and will not discuss patient outcomes or efficacy of the medication.

\section{MethodS}

On November 16, 2020 LRH received a communication regarding the availability of Bamlanivimab, a monoclonal antibody, used in the treatment of mild to moderate COVID-19 infections in high risk individuals to decrease hospitalizations and COVID-19 disease severity and adverse outcomes. This medication received Emergency Use Authorization through the FDA, following the results by Chen, et al which showed preliminary data that Bamlanivimab appeared to increase the speed in which viral RNA declined when compared with placebo. Along with the accelerated decrease in viral RNA, there was a correlated decrease in symptom severity and COVID-19 related hospitalizations in high risk individuals with mild or moderate symptoms when compared to the placebo group. The medication arrived at LRH on November 18,2020 and the first dose was administered during the early morning hours on November 19, 2020 within 24 hours of receiving the medication shipment.

After receiving the notice of intent to make the drug available for our institution, a multi-disciplinary hospital-wide meeting comprised of hospital leadership, emergency department physician leadership, information technology(IT), nursing, pharmacy, patient access, and ambulatory care providers met to determine how we would approach the identification of eligible patients and administration of the medication to the intended patient population. During this meeting it was decided that the Emergency Department(ED) providers at LRH would be best suited to identify the patients and provide the infusion and monitoring of Bamlanivimab treatment. Subsequently the ED leadership team formed a steering group to develop and oversee the new process.

\subsection{Why the Emergency Department?}

The ED was acknowledged as the most suitable setting to identify and subsequently manage this patient population. The ED was chosen for several reasons.

(1) The ED physicians were comfortable identifying patients that required admission vs outpatient therapy for COVID-19 infections.

(2) The ED had a process previously in place for COVID19 testing and disseminating results to discharged pa- tients.

(3) The ED setting was determined to be the most efficient and safest setting where patients could readily be infused with a medication that possessed a risk of anaphylaxis.

(4) There was already a process in place with a designated area within the ED for patients to receive IV placement and infusion therapy as outpatients with monitoring capabilities and staff trained in identifying and treating adverse infusion reactions.

(5) The ED had the necessary equipment for emergent interventions including medications, code cart, and intubation equipment with providers on site that are well trained in emergent interventions.

(6) The ED setting was appropriately set up to isolate COVID-19 positive patients from the non-COVID-19 patient population and adequate personal protective equipment for the providers.

\subsection{Identifying eligible patients}

The steering committee identified three sources of patients for Bamlanivimab therapy.

(1) High risk COVID-19 positive patients who present to the ED for treatment(see Figure 1 for inclusion criteria).

(2) High risk ED patients with a COVID-19 test pending at the time of discharge.

(3) Eligible patients referred to the ED by outpatient hospital and community physicians.

A decision was made to focus on the first two groups in week one and then to roll out to the third group when the process was well established (Discussed under expanding treatment to patients in the community).

\subsection{High risk patients discharged from the ED with COVID test pending}

Utilizing the eligibility criteria published through the FDA emergency use authorization LRH providers were able to identify high risk patients who would meet eligibility for the medication administration (see Figure 1). These patients were identified upon presentation to the ED using clinical diagnosis of COVID-19 infection or by performing COVID-19 testing using one of several COVID tests based on hospital protocol and availability. As one of the eligibility criteria for treatment with Bamlanivimab the patient cannot require hospitalization. ${ }^{[1]}$ The majority of these patients were discharged after initial medical evaluation and COVID-19 testing, while awaiting test results (see Figure 2). There is an average turnaround time of 5 hours for discharge eligible patients. 


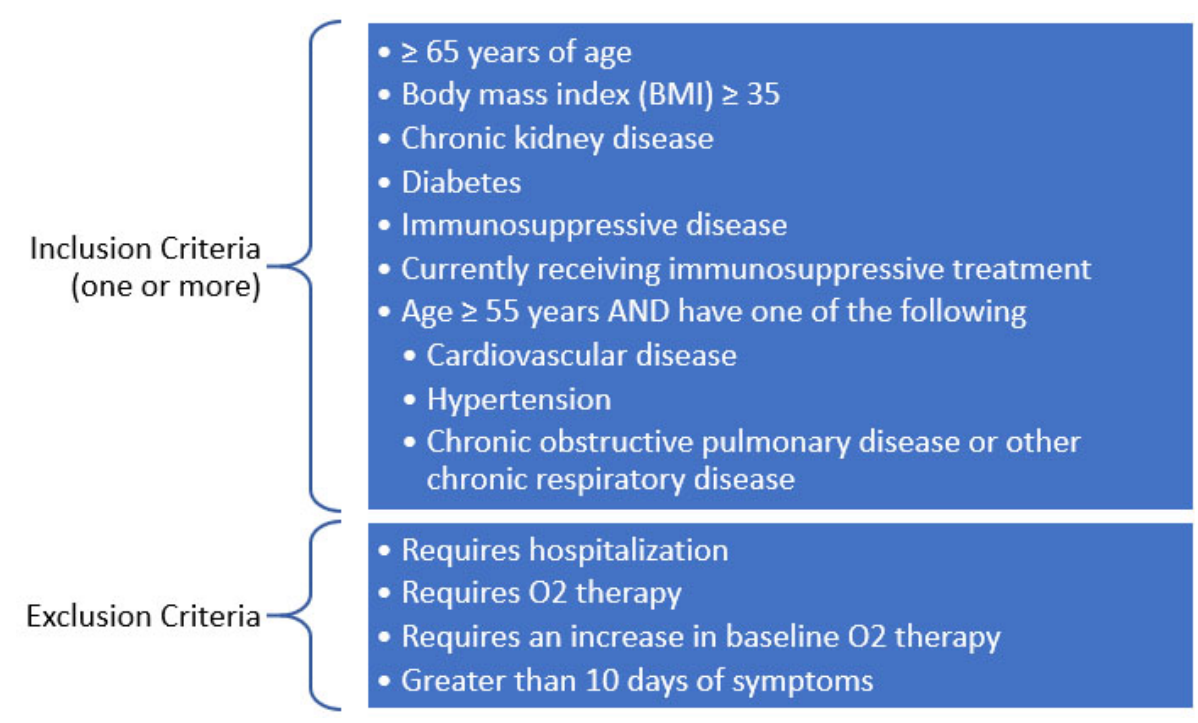

Figure 1. Eligibility criteria

\section{Monoclonal Antibody Treatment in High Risk Patients with Mild to Moderate COVID-19 COVID-19 Status Unknown Emergency Department Lakeland Regional Health}

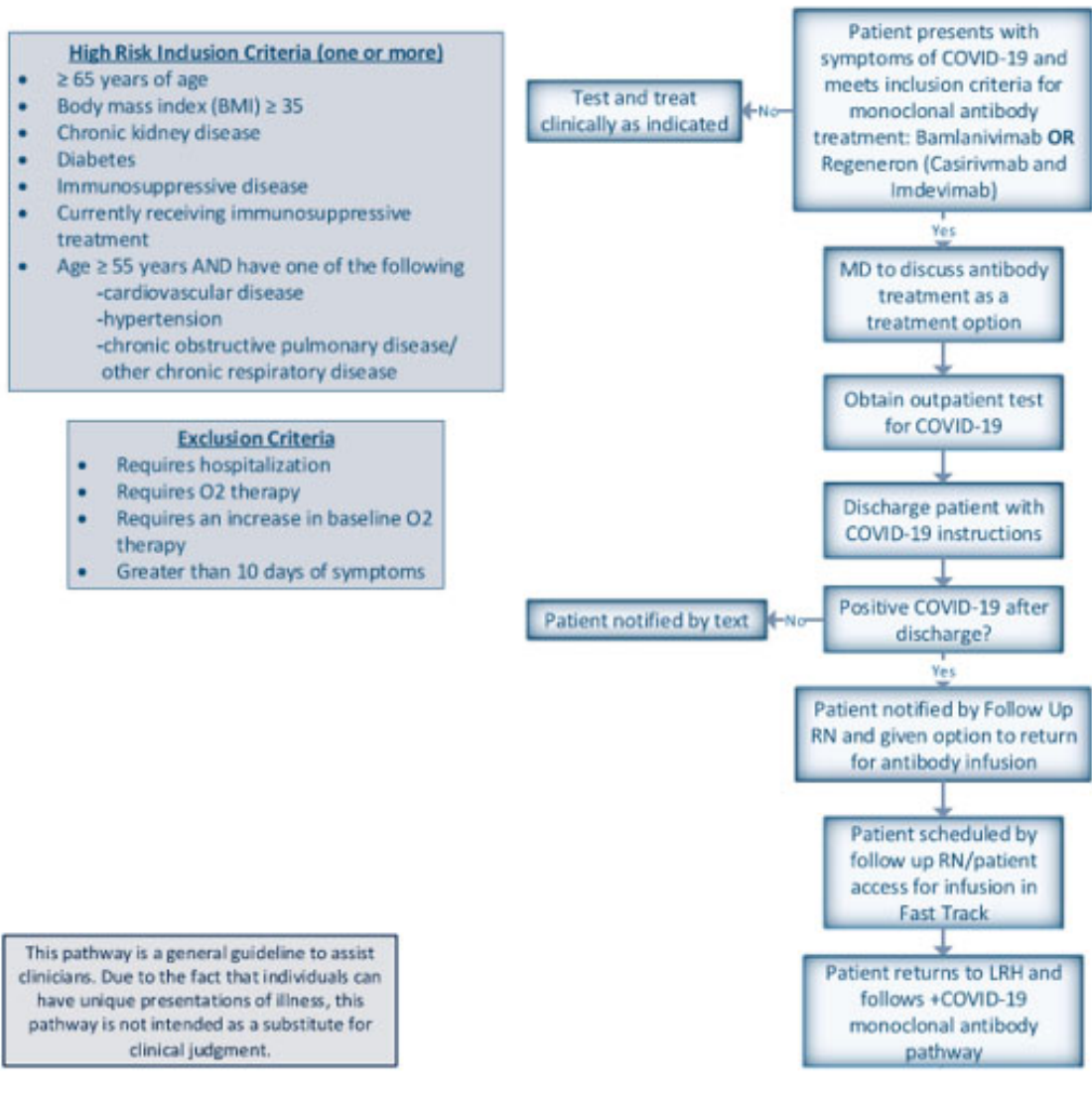

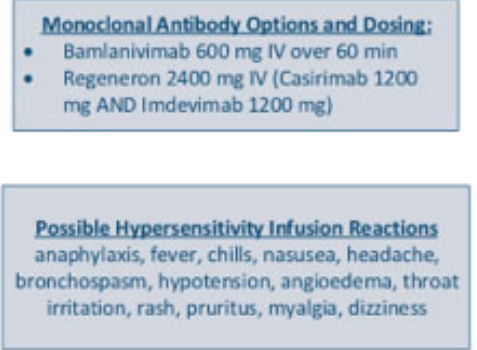

bronchospasm, hypotension, angioedema, throat
irritation, rash, pruritus, myalgia, dizziness

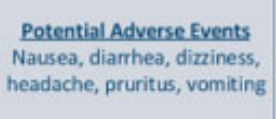

Figure 2. Pathway for COVID+ discharged from the Emergency Department 


\section{Bamlanivimab Adult ED EUA Criteria}

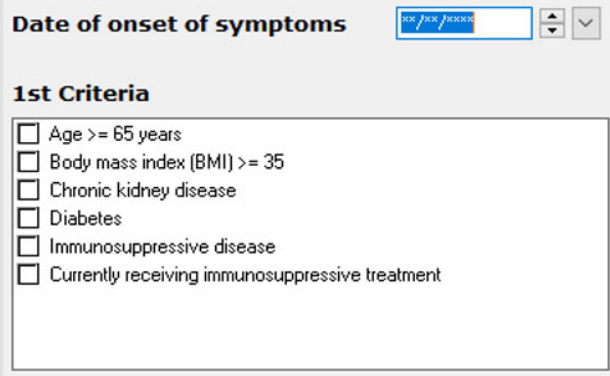

\section{2nd Criteria}

$\square$ Age $>=55$ years AND cardiovascular disease

$\square$ Age $>=55$ years $\mathrm{AND}$ COPD/other chronic respiratory disease

$\square$ Age $>=55$ years AND hypertension

Figure 3. Adult bamlanivimab alert

During the ordering process for COVID-19 testing an alert in the electronic health record (EHR) with the Bamlanivimab eligibility requirements would display on the provider's screen(see Figures 3 and 4). The provider would then mark the patient's risk factors and eligibility criteria. This alert allowed for efficient follow up as discussed below. Discharging stable patients awaiting confirmation of COVID-19 status allowed the ED to turn over beds for additional acute patients and to maintain throughput in a 165 bed ED with a pre-pandemic annual volume of 200,000 patient visits.

During the initial evaluation and again during the discharge process the ED provider and nursing staff would discuss with the patient the possibility of Bamlanivimab infusion if the patient met the predetermined eligibility criteria. These discussions included that they may be contacted to return to the ED for Bamlanivimab infusion if they were to be confirmed COVID-19 positive.

If the COVID-19 test is positive an alert with the patient's information was sent to the ED follow up office through the EHR. The follow up office is comprised of two nurses who have been trained in recognizing results and recalling patients for additional treatment or appointments. Once a positive COVID-19 result is identified the chart is reviewed for Bamlanivimab eligibility as marked by the initial ED provider in the alert window. The follow up office then calls the patient, identifies themselves and the patient, and alerts the patient of the positive COVID-19 test. The nurse follows a script to ensure identification of the patient as a high-risk individual and confirms they meet the eligibility criteria for infusion of Bamlanivimab. If the patient is confirmed eligible the follow up office will then explain the option of receiving Bamlanivimab and the required timeline for effectiveness. If the patient consents to receive Bamlanivimab the follow up office then schedules them for the next available time slot. These time slots are scheduled seven days a week from 11:00 a.m. to 7:00 p.m.

\subsection{Determining setting of medication administration}

For safety and efficiency, it was determined that the first location within LRH that would be fully equipped for this type of infusion and monitoring would be the ED. We began utilizing an area in the "Fast Track" of the ED that was already equipped with cardiopulmonary monitors and was currently being used to facilitate outpatient antibiotic infusions.

\section{Bamlanivimab Treatment for Pediatric Patients Age 12-18}

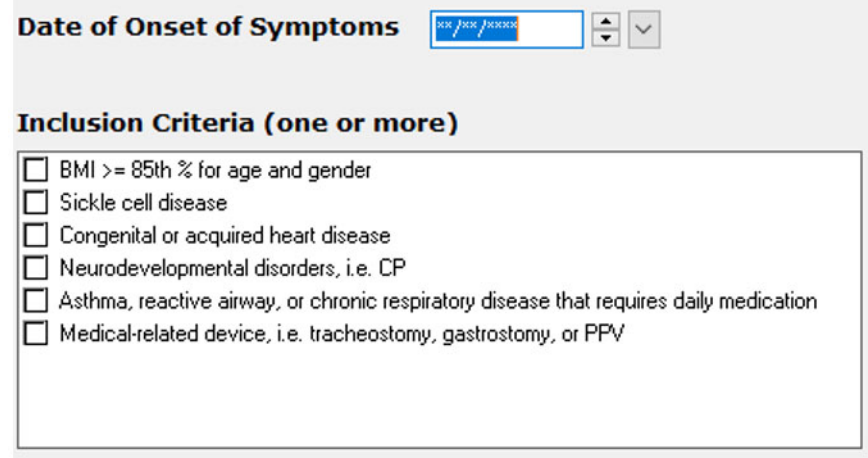

Figure 4. Pediatric bamlanivimab alert 
The patients that were currently utilizing this area for their outpatient infusions were geographically relocated to the pediatric ED as the census within the pediatric ED had seen a significant reduction since the onset of the pandemic. This setting provided available space with monitoring capabili- ties for this population group that needed to isolate away from other patients. This allowed for the continuation of already begun infusion therapies for the community without disrupting patient care or treatment plans.

\section{Monoclonal Antibody Treatment in High Risk Patients with Mild to Moderate COVID-19 Known Positive COVID-19 Emergency Department Lakeland Regional Health}

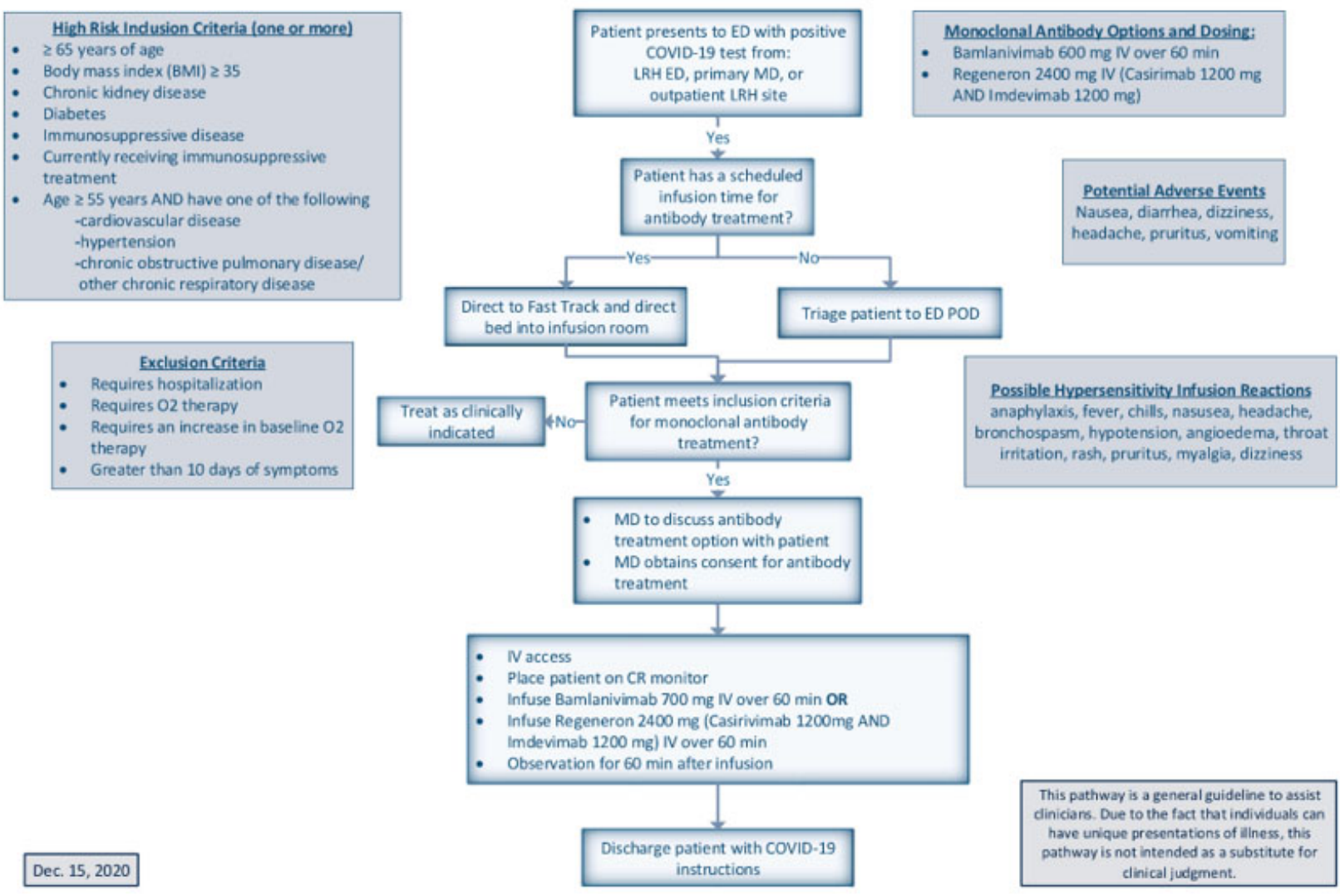

Figure 5. Pathway for COVID+

\subsection{Required equipment and physical space}

As part of the physical setting for the infusion of the Bamlanivimab infusion the space had to be able to handle acute adverse reactions including anaphylaxis. The Fast Track space was already equipped with cardiopulmonary monitoring, a medication dispensing cabinet containing medications for the treatment of allergic reactions, and wall suction. Additional equipment that was placed into this area to ensure immediate availability was oxygen tanks, a code cart, and airway/intubation equipment. Initially patients were placed on stretchers for the infusion and monitoring, however the number of scheduled patients increased, infusion chairs were used. This had the benefit of allowing physical space for additional eligible patients to be scheduled and receive the infusion at the same time, increasing our ability to treat a larger number of individuals at once with the limitation now being the number of available monitors. In the chance of an adverse or allergic reaction requiring intervention occurred, a stretcher was made immediately available and was stored just outside of the infusion space. The infusion space is located in an area where the patients can be isolated from other patients in the treatment area and can be transported into and out of the ED with minimal contact with other patients and staff.

\subsection{Infusion, monitoring, and discharge}

Once the patient arrived for their scheduled infusion, following the pathway, they are registered and placed into the infusion space. The physician then confirms eligibility for monoclonal antibody therapy and along with the nurse ob- 
tains written consent for the treatment. They then place the order for the initiation of monoclonal antibody therapy into the EHR.

Each day, pharmacy premixes the doses of monoclonal antibody therapy to meet the scheduled demand and delivers it the treatment area. The patient receives the medications over one-hour with a one-hour observation period as previously recommended by the FDA's EUA guidelines 1 . Throughout both the infusion and observation periods the patients are on constant cardiopulmonary monitoring with continuous nursing staff present to help recognize any adverse reactions.

If a patient has an adverse or an allergic reaction, the medications is immediately stopped and the patient receives treatment for the adverse reaction. The ED pharmacists then complete the FDA mandated reporting for any adverse reaction. If the infusion is completed with no adverse reaction, the patient is then monitored for an additional hour post infusion and then discharged home (see Figure 5).

\subsection{Expanding treatment to patients identified in the community}

Once we had a well-established pathway and protocol in place for the administration of Bamlanivimab, the hospital recognized that the need of this treatment was far greater than just the population presenting to the ED. With the community in mind, we began to expand the patient pool and include patients from surrounding Polk County and the greater Florida region. This was accomplished in several ways.

Information was sent by the hospital communications office to the entire medical staff, area urgent cares and drive through testing centers educating providers on monoclonal antibody treatment, eligibility criteria and contact information for LRH patient access/central scheduling to get patients enrolled in the system and scheduled. A media press release was also made to the Polk County media in order to increase community awareness that this treatment is available if eligibility criteria is met.

This allowed a secondary pathway for obtaining eligible patients and facilitate treatment on a broader scale. Patients that met criteria and had confirmed testing through their primary care office, drive through testing, or urgent care were now able to be directly scheduled for evaluation and treatment.

If patients present directly to the ED without scheduling an appointment they would be registered and placed within the COVID-19 area in the main ED. They would then be evaluated by the ED provider and confirmed COVID-19 positive via documentation provided by the patient and eligibility criteria would be reviewed. If they meet the eligibility requirements, the patient is educated on monoclonal antibody

Published by Sciedu Press therapy and consented by the physician and nurse and the monoclonal antibody therapy is ordered. These patients then receive the infusion over one hour with an additional onehour monitoring for adverse reactions (see Figure 5). This occurs in the area of the main ED where the patient was initially triaged to and on a cardiopulmonary monitor. This allowed for a larger population of eligible patients to receive this treatment.

\subsection{Adapting to surges}

As the ongoing COVID-19 pandemic continues to surge across the country and within our community we have been able to adapt this protocol as ED resources are pulled to treat a sicker population. With these surges increased resources are required both for the emergent treatment of higher acuity patients but also increased numbers of eligible patients for monoclonal antibody therapy. The most limited resource is space within the ED. When the infusion area is required for the treatment of acute patients or higher numbers of Bamlanivimab eligible patients are scheduled than the existing infusion space allows, the monoclonal antibody therapy is moved to a large conference room adjacent to the ED. With the shift of the treatment area, similar equipment as above is placed into the new space including portable cardiopulmonary monitors, portable suction, oxygen tanks, a code cart, medications utilized in treatment of allergic reactions, infusion pumps and poles, infusion chairs, and a stretcher. Additional nursing staff is dedicated to this area during scheduled infusions for IV placement, infusion, and continued monitoring of the patients. An ED provider is available and evaluates, orders the medications, and discharges the patient as above. Utilizing this space and adapting our protocol we have been able to treat higher numbers of eligible patients with monoclonal antibody therapy even during times where evaluation and treatment of acute patients have required higher resources within the ED and hospital.

\subsection{Efficiency of protocol}

Utilizing this pathway LRH has been able to efficiently identify and treat high risk eligible COVID positive patients. The time frame from the initial notification that this treatment would be available to receiving the first shipment of medication was less than 48 hours and from receipt of the medication until the first use was less than 24 hours. Between the dates of Nov 18, 2020 and February 17, 2021 we have been able to treat a total of 1037 patients with monoclonal antibody therapy including 900 patients with Bamlanivimab and 137 patients with REGN-COV2. This number includes 20 high risk pediatric patients between the ages of 12 and 17 with Bamlanivimab and 5 with REGN-COV2. 


\section{Discussion}

There are many aspects of this protocol that we believe have made the program a success and may be adaptable to other facilities. One of which is the identification of pre-existing resources and pathways and adapting them to the needs of administering monoclonal antibodies. This includes the utilization of the ED in identifying potential treatment candidates, the ED follow up office in confirming positive COVID results and eligibility criteria and scheduling the patients for their infusion, and an infusion area within the ED for both administration of the medication and monitoring. This was able to be accomplished efficiently and facilitated the rapid turnaround time for the initiation of this protocol.

Another major part of this pathway is the application of this protocol within the ED. The ED setting allows for efficient triage of patients who meet the eligibility criteria during the initial patient encounter. The ED setting subsequently provides a safe and efficient transfusion area that requires minimal additional resources except for physical space with cardiopulmonary monitoring capabilities. It also provides a staff that is well versed in proper PPE and emergent interventions in case of a transfusion reaction. Additionally, it allows COVID-19 positive patients to isolate away from a patient population that is otherwise often immunocompromised that is present in outpatient infusion centers, i.e. chemotherapy patients or patients with high risk infections receiving long term IV antibiotic therapy. Finally leveraging the facility's information technology was a critical tool for success and allowed an efficient way to identify and flag eligible patients and schedule the monoclonal antibody therapy.

With this protocol now in place we have the ability to adapt it to additional treatment options as available, including other monoclonal antibody treatment options. Given the potential severity of COVID-19 and complications in high risk patients this protocol was prioritized and resources were devoted to both the formation of the protocol and needs of the treatment pathway.

Not all hospitals have preexisting resources that can be repurposed to allow this rapid utilization and formation of a protocol and treatment. However, it is possible to identify similar resources or create a pathway that best fits within the unique individual institution's capabilities to allow the administration of this treatment in a safe and efficient manner. In our institution utilizing the ED and dedicating additional resources to the ED to assist with this pathway is the most efficient method for delivering large quantities of this treatment to the patient population it is intended to treat.

\section{Conclusion}

This protocol and treatment pathway utilize existing resources to develop a pathway that is safe and efficient and designed to work within our institution. Critical to the development of this protocol was a multi-disciplinary approach through which LRH was able to identify obstacles to the initiation of this therapy, create a protocol, and initiate the proposed pathway within 24 hours of receiving the first shipment of medication. By identifying and utilizing similar resources and pathways available at individual medical centers, it is possible to safely and efficiently treat high risk COVID positive patients with monoclonal antibody therapy on a large scale. Additional data needs to be analyzed regarding the benefits of this treatment, patient outcome, and adverse reactions.

\section{CONFlicts OF INTEREST Disclosure}

The authors declare they have no conflicts of interest.

\section{REFERENCES}

[1] An EUA for Bamlanivimab-A Monoclonal Antibody for COVID19. JAMA. 2020 Dec 11. Epub ahead of print. PMid: 33306087 https://doi.org/10.1001/jama.2020.24415
[2] Chen P, Nirula A, Heller B, et al. SARS-CoV-2 Neutralizing Antibody LY-CoV555 in Outpatients with COVID-19. NEJM. 2020. PMid: 33113295. https://doi.org/10.1056/NEJMoa2029849 